\title{
Antenna Selection and Power Allocation in Massive MIMO
}

\author{
Paranche Damodaran SELVAM, Kuttathati Srinivasan VISHVAKSENAN \\ Dept. of ECE, SSN College of Engineering, Kalavakkam 603110, Tamil Nadu, India \\ paranche@eurecom.fr, vishvaksenanks@ssn.edu.in
}

Submitted August 21, 2018 / Accepted November 15, 2018

\begin{abstract}
This paper explores the massive multiple-input multiple-output (Ma-MIMO) communication system possessing large number of antennas at the basestation (BS) serving multiple user terminal (UT) in single cell configuration. Due the large number of antennas at the BS, the RF chains used is also increasing with the increase in the total power consumption of the system such as circuit power consumption, filter, mixer and digital to analog converter power consumption. The main aim of this paper is to reduce the transmit power consumption with the proposed antenna selection and power allocation approach. Initially, the equal power is allocated to users to find the optimal number of antenna selection. Then for the number of antennas selected the optimal power allocation is derived to users. An algorithm is proposed to iteratively find the antenna selection and power allocation. The simulation is done to evaluate the average data rate and to find the optimal transmit power of the system.
\end{abstract}

\section{Keywords}

Massive MIMO, antenna selection, power allocation

\section{Introduction}

The Massive Multiple Input Multiple Output (Ma-MIMO) antenna is a multi-user MIMO that is used to massively make better use of spectral efficiency, robustness to interference, increased bandwidth and reduced latency and it is used to provide a solution for high demand communication system like cellular mobile communication that require high data rate, less interference and better coverage and connectivity. The channel between the transmitter and receiver is estimated from orthogonal pilot sequences which are limited by the coherence time of the channel. Ma-MIMO has some main issues that are related to the technique used in the antenna selection and power allocation. We provide an overview of recent trends concerning antenna selection and power allocation then provide an enhancement to selected technique.

In [1], an antenna selection technique has been reviewed with considering different criterion such that channel capac- ity and bit error rate seeking both cost and hardware complexity reduction and It has proven that the performance is improved with the increase of number of receive antennas. The main target is to improve the spectral and transmit-energy efficiency [1-7] and it is obtained by increasing the number of antennas and RF chains. However, system complexity and hardware energy consumption is increased with the reduced number of RF chains in the antenna selection process. A near optimal convex optimization based on selection used as a reference. The sum-rate achieved is evaluated with a simple method of selecting antennas with the highest received power. In this power-based antenna selection method for the measured channels, the performance close to the convex optimization scheme is achieved. The same objective was in [4], [7-12] but it used the norm-and-correlation-based selection algorithm with low complexity for energy efficiency maximization has been proposed. Selection metric considers the effect of the norm of each channel column and correlation between columns while attaining low computational complexity. While in [6] the problem of joint multicast beam forming and antenna selection for multiple co-channel multicast groups was the motivation of the work and the proposed algorithm has achieved a reduction in a number of required antennas that are essential to produce the wanted signal level along with less transmission power. The performance is satisfactory and has presented less complexity along with improvement in other attributes.

Another point of interest has been studied in [4,9,13-15] which is accurate transmit-side channel state information (CSI) and a new algorithm named antenna group scheduling (AGS) algorithm which combines antenna selection and user scheduling for pursuing a further reduction in feedback overhead has been proposed. The AGS was built to enhance channel along with selecting the best antennas based on the best gain obtained. The Simulation has proven that the proposed technique fulfill an impressive feedback overhead reduction over the conventional beamforming techniques (33\%-50\%) within the same target sum rate needs. In [1], [6], MIMO can improve the spectral efficiency (SE) jointly with the energy efficiency (EE). Antenna selection process, that is necessary for Ma-MIMO system in both transmitting and receiving end. In [1], [16], some algorithms "Particle Swarm Opti- 
mization(PSO), Genetic Algorithm (GA), and Artificial Bee Colony (ABC)" has been used and compared and they are used to achieve better antenna selection with higher capacity and less CPU time. In [12,13,17], the paper has focused its interest in the channel state information (CSI), acquiring the CSI plays a central role to provide high system performance. An efficient transmit antenna selection technique acquiring the amount of the required CSI for a Ma-MIMO system in the broadcast channel has been used. The method is to use receiver chosen channel and feedback the selection to the transmitter in form of a feedback till all receiver finish then the transmitter will determine which antennas will be used and which are to be neglected. The objective is to maximize the data rates. The optimal solution is a highly complex exhaustive brute force search (BFS) [10], [18] over all possible combinations of antennas and users. The proposed algorithm aims to maximize the achievable sum-rate and to make use of both the spatial selectivity gain and multiuser diversity gain offered by the antenna selection and user scheduling, respectively. The key idea of the proposed algorithm was to successively eliminate both undesired transmit antennas. The proposed algorithm is able to achieve near-optimal performance with low computational complexity. Spatial multiplexing emerged from the fact that in a rich scattering environment, it is possible for the receiver to descramble signals that are transmitted simultaneously from the multiple antennas. Thus one is able to send parallel independent data streams and achieve overall capacity. In [12], the proposed antenna selection is based on the idea of lowering the cost, size and the power consumption by ranking the antennas based on their channel gain to all mobile stations. Then the transmitter computes the downlink sum rate with $\mathrm{S}$ (assumed the antenna to be used) consecutive antenna elements, the transmitter selects the $S$ that is giving the highest sum-rate, the selected $S$ antennas are used to transmit and connect to $\mathrm{n}$ users however the rest are turned off for that time slot. The proposed antenna selection technique provides better and less computation. In [4], [18-20], a low complexity greedy user selection scheme with linear zero forcing pre-coding at the base station has been proposed. The complexity of the proposed algorithm is remarkably low i.e. $\mathrm{O}(\mathrm{MK})$ while the performance is same as that of the best greedy algorithm known so far. According to previous approaches consider the issue of transmit antenna choice for Ma-MIMO systems by maximizing the determinant modulus of the chosen channel matrix [21]. In [11], the main objective is to develop a practical solution for the complexity of RF switching by introducing binary switching system that performs the antenna selection techniques and reduces system cost and complexity. Antenna selection with respect to the system capacity and reasonable signal-to-noise ratio has been investigated [22-25] also the effect of antenna selection techniques on the coding system and the channel capacity itself even if the channel capacity is one of the criterion. In Tab. 1 antenna selection and power allocation related work is summarized.

\begin{tabular}{|l|l|}
\hline Work area & Key points \\
\hline \multirow{5}{*}{ Antenna Selection } & $\begin{array}{l}\text { norm-and-correlation-based se-lection algo- } \\
\text { rithm with low complexity for energy effi- } \\
\text { ciency maximization [4], [7-12] }\end{array}$ \\
\cline { 2 - 2 } & $\begin{array}{l}\text { transmit-side channel state information (CSI) } \\
\text { used for antenna selection and user schedul- } \\
\text { ing to reduce feedback overhead [4,9, 13-15] }\end{array}$ \\
\cline { 2 - 3 } & $\begin{array}{l}\text { PSO, GA, and ABC used and compared and } \\
\text { they are used to achieve better antenna se- } \\
\text { lection with higher capacity and less CPU } \\
\text { time [1], [16]. }\end{array}$ \\
\hline \multirow{2}{*}{ Power allocation } & $\begin{array}{l}\text { equal power allocation to user [3, 15,26] } \\
\text { various power consumption factor considered } \\
\text { apart from the transmit power required at the } \\
\text { BS [10], [26] }\end{array}$ \\
\hline
\end{tabular}

Tab. 1. Related work in antenna selection and power allocation.

In this work the antenna selection and power allocation is considered in order to maximize the achievable sum rate and reduce the total power consumption by the RF chains. The main contribution of this work is elaborated below:

- For a single cell Ma-MIMO downlink system with multi-user, an efficient antenna selection and power allocation is proposed to evaluate the achievable rate under the limited number of RF chains.

- In the first step equal power is allocated to user to simplify the problem proposed later, variable power is allocated to user based on the proposed Algorithm I given in Sec. 2.3.

- Simulation results are provided for the proposed system to demonstrate the performance of antenna selection and power allocation in the single cell multi-user Ma-MIMO communication system.

The paper is organized as follows. In Sec. 2, the MaMIMO downlink system model is elaborated with power consumption model. The problem formulation of antenna selection and power allocation is done in this section. Also, the proposed joint antenna selection and power allocation is explained in this section. In Sec. 3, the numerical results are evaluated. In Sec. 4, the conclusion is presented.

\section{System Model}

In this paper single cell Ma-MIMO system is considered serving multi-users with each user having single antenna. The base station (BS) is having $N$ antennas serving the $K$ users sharing the resource block belonging to same time-frequency unit. At the BS, the assumption of perfect channel state information (CSI) is made. The BS performs antenna selection among the available $M$ antennas choosing $N$ best antennas in the cell and also allocate power to the users. The BS performs jointly the antenna selection and power allocation. The signal received at the $k^{\text {th }}$ user is given by [1-7] 


$$
\mathbf{y}_{\mathrm{dl}}=\sqrt{\mathbf{T}} \mathbf{B x}+\mathbf{n} .
$$

The signal vector $\mathbf{x}$ at $\mathrm{BS}$ is written as

$$
\mathbf{x}=\sqrt{\psi} \mathbf{P s}
$$

where

$$
\begin{gathered}
\boldsymbol{y}_{\mathrm{dl}}=\left[y_{\mathrm{dl}, 1}, y_{\mathrm{dl}, 2}, \cdots, y_{\mathrm{dl}, k}\right]^{\mathrm{T}}, \\
\mathbf{T}=\operatorname{diag}\left(\mathbf{t}_{\mathrm{d}}\right), \quad \mathbf{t}_{\mathrm{d}}=\left[t_{\mathrm{d}, 1}, t_{\mathrm{d}, 2}, \cdots, t_{\mathrm{d}, k}\right]^{\mathrm{T}}, \\
\mathbf{b}_{\mathrm{k}}=\left[b_{\mathrm{k} 1}, b_{\mathrm{k} 2}, \cdots, b_{\mathrm{k} M}\right], \quad b_{k m}, \quad 1 \leq k \leq K, 1 \leq m \leq M, \\
\boldsymbol{n}=\left[n_{1}, n_{2}, \cdots, n_{K}\right]^{\mathrm{T}}, \quad n_{k} \sim C \mathcal{N}(0,1), \\
\boldsymbol{x}=\left[x_{1}, x_{2}, \cdots, x_{M}\right]^{\mathrm{T}}, \\
\mathbf{P}=\left[p_{1}, p_{2}, \cdots, p_{k}\right], \\
\boldsymbol{s}=\left[s_{1}, s_{2}, \cdots, s_{K}\right]^{\mathrm{T}}, \\
\mathbb{E}\left\{\left|s_{k}\right|^{2}\right\}=1 .
\end{gathered}
$$

The received signal at the $k^{\text {th }}$ user is denoted by $\boldsymbol{y}_{\mathrm{dl}}$. $\mathbf{T}$ indicates the available transmit power in the downlink for the user. The downlink channel matrix is denoted by $\mathbf{B}$ with the dimension $K \times M$. The channel matrix elements are represented by $b_{k m}, 1 \leq k \leq K, 1 \leq m \leq M$ indicates the channel elements from $m^{\text {th }}$ transmit antenna at BS to $k^{\text {th }}$ user. $b_{k m}$ is defined as the independent and identically distributed (i.i.d) complex Gaussian random variables with zero mean and variance $\zeta_{k}, b_{k m} \sim C \mathcal{N}\left(0, \zeta_{k}\right)$, where $\zeta_{k}$ denotes the large-scale fading. $\mathbf{n}$ denotes noise vector signal with complex Gaussian having zero mean and unit variance $n_{k} \sim C \mathcal{N}(0,1) . \quad x$ indicates signal transmitted from BS to user. The precoding matrix is denoted by $\mathbf{P}$ having the matrix dimension $M \times K$. $\mathbf{s}$ is the symbol transmitted to user from the BS and it satisfies the condition $\mathbb{E}\left\{\left|s_{k}\right|^{2}\right\}=1 . \psi$ denotes a constant which satisfies the condition $\mathbb{E}\left\{\|\mathrm{x}\|^{2}\right\}=1$ and is defined as $\psi=\frac{1}{\mathbb{E}\left\{\operatorname{tr}\left(\mathbf{P}^{\mathrm{H}} \mathbf{P}\right)\right\}}$.

The signal at the receiver can be rewritten by substituting (2) into (1) as

$$
\mathbf{y}_{\mathrm{dl}}=\sqrt{\psi \mathbf{T}} B \mathrm{Ps}+\mathbf{n} .
$$

For Ma-MIMO with single cell configuration the $k^{\text {th }}$ user received signal is written as

$$
\begin{gathered}
\mathbf{y}_{\mathrm{dl}, k}=\sqrt{\psi t_{\mathrm{d}}} \mathbf{b}_{k} \mathbf{P s}+n_{k}, \\
y_{\mathrm{dl}, k}=\underbrace{\sqrt{\psi t_{\mathrm{d}}} \mathbf{b}_{k} \mathbf{p}_{k} s_{k}}_{\text {desiredsignal }}+\underbrace{\sqrt{\psi t_{\mathrm{d}}} \sum_{i=1, i \neq k}^{K} \mathbf{b}_{k} \mathbf{p}_{i} s_{i}}_{\text {inter-UT interference }}+\underbrace{n_{k}}_{\text {noise }} .
\end{gathered}
$$

The received SINR for user is expressed as

$$
\operatorname{SINR}_{k}=\frac{\psi t_{\mathrm{d}}\left|\mathbf{b}_{k} \mathbf{p}_{k}\right|^{2}}{\psi t_{\mathrm{d}} \sum_{i=1, i \neq k}^{K}\left|\mathbf{b}_{k} \mathbf{p}_{i}\right|^{2}+1}
$$

The downlink rate for the $k^{\text {th }}$ user is expressed based on the Shannon's formula is given below

$$
R_{k}=\log _{2}\left(1+\operatorname{SINR}_{k}\right)
$$

The total sum rate of $K$ users is given as

$$
R_{\text {sum }}=\sum_{k=1}^{K} \mathbb{E}\left\{R_{k}\right\}
$$

Based on (6) and (8) the sum rate is rewritten as

$$
R_{\text {sum }}=\sum_{k=1}^{K} \mathbb{E}\left\{\log _{2}\left(1+\frac{\psi t_{\mathrm{d}}\left|\mathbf{b}_{k} \mathbf{p}_{k}\right|^{2}}{\psi t_{\mathrm{d}} \sum_{i=1, i \neq k}^{K}\left|\mathbf{b}_{k} \mathbf{p}_{i}\right|^{2}+1}\right)\right\} .
$$

\subsection{Power Consumption Model}

The circuit power consumption for transmit antenna, power required for signal processing and cooling loss are the key power consumption factor apart from the transmit power required at the BS [10], [26]. The power consumption model can be written as

$$
t_{\mathrm{sum}}=\mu\left(\frac{K t_{\mathrm{d}}}{\rho}+t_{\mathrm{cir}}+t_{\mathrm{sta}}\right)
$$

where $\mu$ represents the loss factor of system power supply related to main system, DC system and cooling system. The efficiency of the power amplifier (PA) is represented by $\rho$. The power consumption of the circuits related to RF chains is represented by $t_{\mathrm{cir}}$. The static power which is independent of transmit antenna number is given by $t_{\text {sta. }}$. The $t_{\text {cir }}$ is modeled as

$$
t_{\mathrm{cir}}=M\left(t_{\mathrm{dac}}+t_{\mathrm{mix}}+t_{\mathrm{filt}}\right)+t_{\mathrm{syn}}
$$

where $t_{\mathrm{dac}}$ is the power consumed by the DAC circuit, $t_{\text {mix }}$ represents power consumed in the mixer, $t_{\text {filt }}$ represents power consumed in the filter and $t_{\text {syn }}$ represents power consumed in the frequency synthesizer. If the parameters in (11) considered as constant, except $t_{\mathrm{d}}$ and $\mathrm{M}$ then the $t_{\text {sum }}$ increases monotonically with respect to $M$. The circuit power consumption is given below if $t_{\max }$ is the maximum available power at the $\mathrm{BS}$ as

$$
\begin{gathered}
t_{\text {out }}+\sum_{n=1}^{N} \alpha_{n} t_{\text {cir }} \leq t_{\max }, \\
t_{\text {out }}=\sum_{k=1}^{K} t_{k},
\end{gathered}
$$

$$
\alpha_{n}=\left\{\begin{array}{l}
1, \text { antenna } \mathrm{n} \text { is selected } \\
0, \text { otherwise }
\end{array}\right.
$$

where $t_{\text {out }}$ is the output transmitted power. $\alpha_{n}$ is defined as antenna selection coefficient [3]. 


\subsection{Problem Formulation}

The optimal solution is to find the power allocation in the transmitting antenna among the selected transmit antenna. The optimal number of antennas and power allocation have to be obtained jointly to attain the maximum capacity. The sum capacity of the Ma-MIMO system is described by the following equation $[3,19,27]$

$$
\mathbf{C}(\mathbf{B})=\max _{\mathbf{T} \in \mathcal{T}} \log _{2} \operatorname{det}\left(\mathbf{I}_{M}+\rho \mathbf{B}^{\mathrm{H}} \mathbf{T B}\right)
$$

where, $\mathcal{T} \subseteq \mathbb{R}^{K \times K}$ is the set of non-negative diagonal matrices with $\operatorname{Tr}[\mathbf{T}]=1$. If the antenna selection is employed with a subset of antennas at the BS, the sum capacity is written as function antenna selection is given as

$$
C\left(\mathbf{B}_{[\mathcal{N}]}\right)=\max _{\mathbf{T} \in \mathcal{T}} \log _{2} \operatorname{det}\left(\mathbf{I}_{N}+\rho \mathbf{B}_{[\mathcal{N}]}^{\mathrm{H}} \mathbf{T} \mathbf{B}_{[\mathcal{N}]}\right) .
$$

By solving the following problem, the optimal capacity can be determined for antenna selection and power allocation for all users

$$
C(\mathbf{B})=\max _{\substack{\mathbf{T} \in \mathcal{T},|\mathcal{N}|=N}} \log _{2} \operatorname{det}\left(\mathbf{I}_{N}+\rho \mathbf{B}_{[\mathcal{N}]}^{\mathrm{H}} \mathbf{T B}_{[\mathcal{N}]}\right) .
$$

Since the optimization problem is non-convex, the antenna selection and power allocation must be jointly designed. The problem can be partially simplified by allocating equal power to users by the predetermined power allocation matrix $\mathbf{T}=\frac{1}{K} \mathbf{I}_{K}$. Under the equal power allocation regime the optimization problem becomes

$$
C(\mathbf{B})=\max _{|\mathcal{N}|=N} \log _{2} \operatorname{det}\left(\mathbf{I}_{N}+\rho \mathbf{B}_{[\mathcal{N}]}^{\mathrm{H}} \mathbf{T} \mathbf{B}_{[\mathcal{N}]}\right) .
$$

Though, this is a simplified form, the channel matrix is tantamount to solving a subset selection

$$
\mathcal{N}^{*}=\arg \max _{|\mathcal{N}|=N} \log _{2} \operatorname{det}\left(\mathbf{I}_{N}+\rho \mathbf{B}_{[\mathcal{N}]}^{\mathrm{H}} \mathbf{T} \mathbf{B}_{[\mathcal{N}]}\right) .
$$

The problem can be solved by convex optimization but having the complexity using the $O\left(M^{3.5}\right)$. For the Ma-MIMO with hundreds of antenna the computation complexity is highly expensive. It is desirable to choose an approach with complexity increasing linearly with $M$.

The power consumed in the circuit when all the antennas activated could not produce maximum sum rate. The critical problem is to find an optimal solution with antenna selection and power allocation among the users. The problem formulated over all realizations for the average sum rate can be written as

$$
\begin{aligned}
\underset{\mathbf{t}, \alpha_{n}}{\operatorname{maximize}}{ }_{n=1 \ldots N} & R_{\mathrm{avg}}=\mathbf{E}\left\{\sum_{k=1}^{K} R_{k}\right\} \\
\text { subject to } & \sum_{k=1}^{K} t_{k}+\sum_{n=1}^{N} \alpha_{n} t_{\mathrm{c}} \leq t_{\mathrm{max}}, \\
& \sum_{n=1}^{N} \alpha_{n} \geq K, \\
& \alpha_{n} \in\{0,1\}, n=1 \ldots N .
\end{aligned}
$$

The problem formulation is non-convex and can not be solved using water filling algorithm for optimal power allocation for users. Due to the multi-user interference the problem becomes non-convex [26]. The transmitter performs the joint operation of antenna selection selecting $N$ antennas among the $M$ antennas and power allocation.

\subsection{Joint Antenna Selection and Power Allocation}

Initially, in the first case equal power allocation is considered in this section in order to simplify the problem and later power allocated for various user based on the approach adopted. The simplified problem is to find the number of antennas that produce maximum sum-rate from the available $\mathrm{m}$ antennas. The conjugate precoding is adapted to reduce the complexity of the beamforming. The asymptotic sum-rate is approximated in [26] for the conjugate precoding. Under the multi user interference the term can be approximated by its expectation as

$$
\frac{1}{K} \sum_{i=1, i \neq k}^{K}\left|\mathbf{b}_{\mathbf{k}} \mathbf{b}_{\mathbf{i}}^{\mathrm{H}}\right|^{2} \approx \mathbf{E}\left\{\left|\mathbf{b}_{\mathbf{k}} \mathbf{b}_{\mathbf{i}}^{\mathrm{H}}\right|^{2}\right\}=\sum_{n=1}^{N} \alpha_{n}
$$

The average $\mathrm{SINR}_{\mathrm{avg}, k}$ for any user over the channel realizations can be approximately written as

$$
\mathrm{SINR}_{\mathrm{avg}, k} \rightarrow \frac{\frac{t_{\mathrm{out}}}{K} \sum_{n=1}^{N} \alpha_{n}}{t_{\mathrm{out}}+K}
$$

For all the users $\mathrm{SINR}_{\mathrm{avg}, k}$ is same. The average sum-rate can be obtained as a function of $t_{\max }$, the fixed consumed power $t_{\mathrm{c}}$, the number of users $K$, and the number of selected antennas

$$
R_{\mathrm{avg}}=K \log _{2}\left(1+\frac{\sum_{n=1}^{N} \alpha_{n}\left(t_{\mathrm{max}}-t_{\mathrm{c}} \sum_{n=1}^{N} \alpha_{n}\right)}{K\left(t_{\max }-t_{\mathrm{c}} \sum_{n=1}^{N} \alpha_{n}+K\right)}\right) .
$$

The $R_{\text {avg }}$ becomes a concave function because the second order derivative of average SINR is negative. The optimal number of selected antenna is given as

$$
\mathcal{N}^{*}=\operatorname{argmax}_{N}\left(R_{\mathrm{avg}}\right), 0<\mathcal{N}^{*}<t_{\max } / t_{\mathrm{c}}
$$

where $t_{\max } / t_{\mathrm{c}}$ is the maximum number of antennas that can be supported by the system due to circuit power constraint. Then, the optimal number of antenna selection is derived as

$\mathcal{N}^{*}= \begin{cases}\lfloor g\rfloor & \text { if } R_{\mathrm{avg}}(\lfloor g\rfloor)>R_{\mathrm{avg}}(\lceil g\rceil) \text { or }\lfloor g\rfloor=\left\lfloor t_{\mathrm{max}} / t_{\mathrm{c}}\right\rfloor \\ \lceil g\rceil & \text { otherwise }\end{cases}$

where

$$
g=\frac{t_{\max }+K-\sqrt{K\left(t_{\max }+K\right)}}{t_{\mathrm{c}}}<t_{\max } / t_{\mathrm{c}}
$$


The optimal antenna selection allows to determine the amount of power consumed at the RF chains. The transmitted power for $k^{\text {th }}$ user can be expressed as

$$
t_{k}=\frac{t_{\max }-N^{*} \cdot t_{\mathrm{c}}}{K}
$$

In the second case, the system sum-rate is derived for the joint optimal antenna selection and power allocation among users. An algorithm is proposed to maximize the sum-rate by iterative approach selecting a randomly chosen antennas from fixed number of antennas and optimally allocating power to users. The algorithm is terminated when the sum-rate starts decreasing. The optimal number of antenna selection is estimated with the respective optimal power allocation.

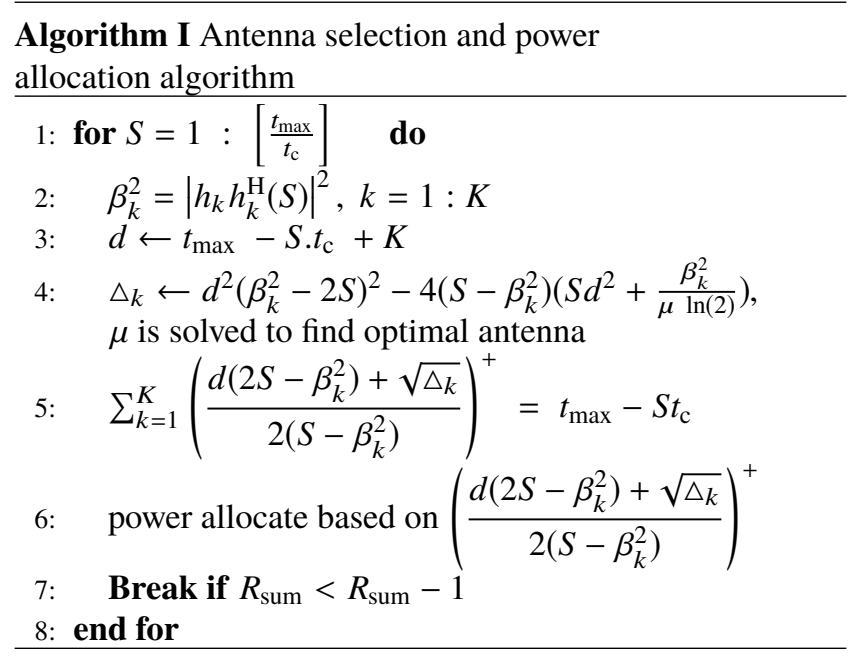

The power allocation is not equal among users, let us consider the multi-user interference as $\left|\mathbf{b}_{\mathbf{k}} \mathbf{b}_{\mathbf{i}}^{H}\right|^{2}=\gamma_{\mathbf{k}}^{2}$ and desired signal $\left|\mathbf{b}_{k} \mathbf{b}_{k}^{\mathrm{H}}\right|^{2}=\beta_{k}^{2}$. The interference term can be approximated as in [26]

$$
\begin{gathered}
\sum_{i=1, i \neq k}^{K} \frac{t_{i}}{\eta}\left|\mathbf{b}_{\mathbf{k}} \mathbf{b}_{\mathbf{i}}^{\mathrm{H}}\right|^{2} \rightarrow \mathbf{E}\left\{\sum_{i=1, i \neq k}^{K} \frac{t_{i}}{\eta}\left|\mathbf{b}_{\mathbf{k}} \mathbf{b}_{\mathbf{i}}^{\mathrm{H}}\right|^{2}\right\} \\
\sum_{i=1, i \neq k}^{K} \frac{t_{i}}{\eta}\left|\mathbf{b}_{\mathbf{k}} \mathbf{b}_{\mathbf{i}}^{\mathrm{H}}\right|^{2}=\sum_{i=1, i \neq k}^{K} \frac{t_{i}}{K}
\end{gathered}
$$

where, the $k^{\text {th }}$ user $\operatorname{SINR}_{\mathrm{avg}, k}$ is written as

$$
\mathrm{SINR}_{\mathrm{avg}, k}=\frac{t_{k} \beta_{k}^{2}}{S\left(t_{\max }-S t_{\mathrm{c}}-t_{k}+K\right)} .
$$

The optimization problem for joint antenna selection and power allocation in (8) can be written as

$$
\begin{array}{ll}
\underset{T}{\operatorname{maximize}} & R=\sum_{k=1}^{K} \log _{2}\left(1+\frac{t_{k} \beta_{k}^{2}}{S\left(t_{\max }-S t_{\mathrm{c}}-t_{k}+K\right)}\right) \\
\text { subject to } & \sum_{k=1}^{K} t_{k}+S t_{\mathrm{c}} \leq t_{\max } .
\end{array}
$$

The above optimization problem can be solved by using the Lagrangian operation and can be written as

$$
\begin{array}{r}
L\left(\left\{t_{k}\right\}, \mu\right)=\sum_{k=1}^{K} \log _{2}\left(1+\frac{t_{k} \beta_{k}^{2}}{S\left(t_{\max }-S t_{\mathrm{c}}-t_{k}+K\right)}\right) \\
+\mu\left(\sum_{k=1}^{K} t_{\mathrm{k}}+S t_{\mathrm{c}}-t_{\max }\right) .
\end{array}
$$

The above optimization can be solved by taking derivative with respect to $t_{k}$

$$
t_{k}^{2}\left(S-\beta_{k}^{2}\right)+t_{k} d\left(\beta_{k}^{2}-2 S\right)+S d^{2}+\frac{\alpha \beta_{k}^{2}}{\ln (2) \mu}=0
$$

where $d=t_{\max }-S t_{\mathrm{c}}+K$.

The Algorithm I solves the (36) for antenna selection and the respective power allocation to the users. The algorithm converge at the point where the sum-rate shows the decrease in value. The concave nature of sum-rate, there exists a convergence point to the algorithm resulting in the maximum sum-rate achieved with the optimal solution.

\section{Numerical Evaluation}

In this section, the numerical simulation performance is evaluated for the proposed antenna selection and power allocation in Ma-MIMO system. The simulation parameters are shown in the Tab. 2. For the Ma-MIMO system at the BS the number of antennas installed is 400 and the total number of user is 10. Each user is having 2 antennas at the user terminal. The cell radius is considered $250 \mathrm{~m}$. the distance between the BS and UT is $35 \mathrm{~m}$. The coherence block length is 200. The maximum transmit power at BS is $35 \mathrm{dBm}$. Under these simulation parameters the system performance is evaluated and illustrated in Figs. 1-4.

\begin{tabular}{ll}
\hline Parameters & Value \\
\hline Number of antennas at BS & 400 \\
Number of user treminals & 10 \\
Number of antennas at user terminals & 2 \\
Number of channel realizations & 10 \\
Total channel bandwidth & $10 \mathrm{MHz}$ \\
Carrier frequency & $2 \mathrm{GHz}$ \\
Number of subcarriers & 600 \\
Subcarrier bandwidth & $30 \mathrm{kHz}$ \\
Cell radius & $250 \mathrm{~m}$ \\
Maximum transmit power & $35 \mathrm{dBm}$ \\
Noise Figure & $5 \mathrm{~dB}$ \\
Receiver noise density & $-101 \mathrm{dBm} / \mathrm{Hz}$ \\
Minimal user distance from base station & $35 \mathrm{~m}$ \\
Coherence block length & 200 \\
Penetration loss & $20 \mathrm{~dB}$ \\
\hline
\end{tabular}

Tab. 2. Simulation parameters. 


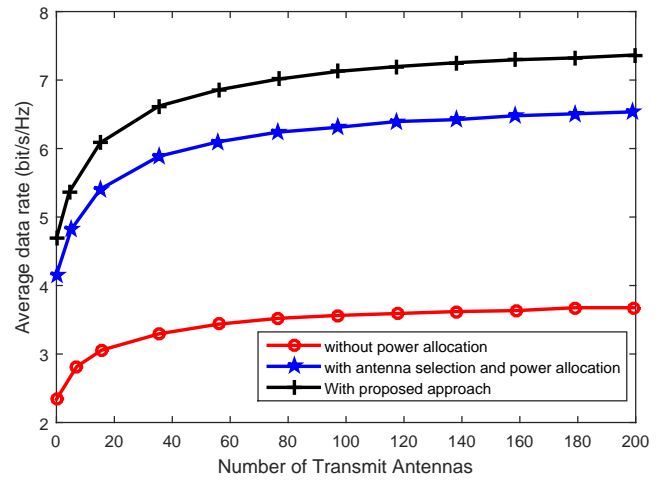

Fig. 1. Performance of average data rate versus number of transmit antennas.

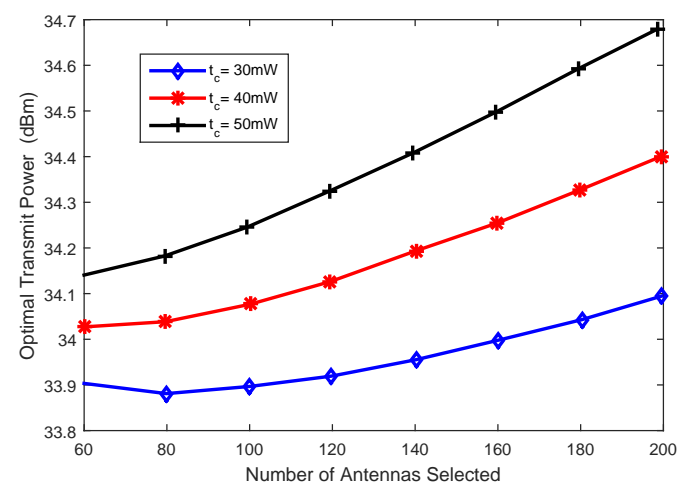

Fig. 2. The total sum rate performance for antenna selection with power allocation scheme.

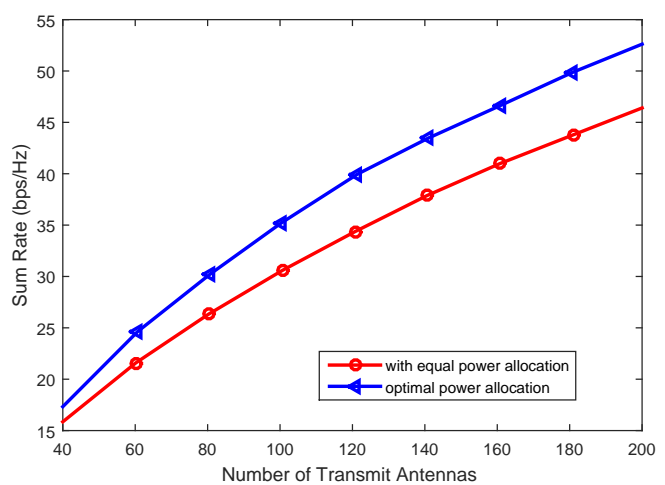

Fig. 3. The performance of optimal transmit power with antenna selection under various circuit power levels.

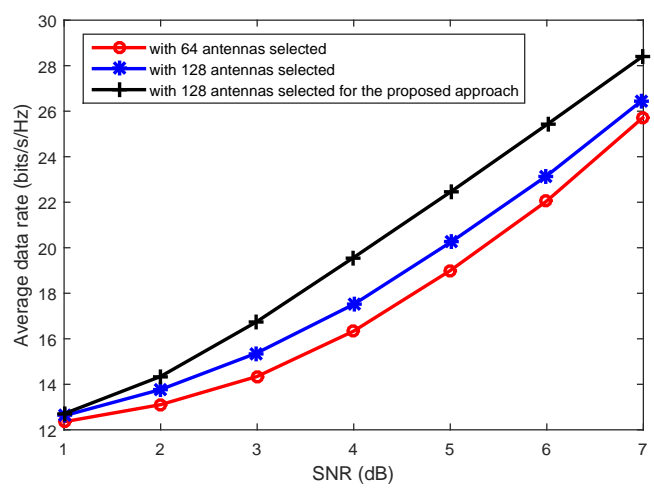

Fig. 4. The performance of SNR vs average data rate for 128 antennas selected.
In Fig. 1, the average data rate versus number of transmit antenna performance is illustrated for the proposed antenna selection and power allocation approach. With the proposed approach the average data rate is improved compared to the other antenna selection and power allocation approach.

In Fig. 2, the sum rate performance of antenna selection with equal power allocation and with optimal power allocation is shown. With the optimal power allocation and antenna selection the total sum rate performance is improved.

Figure 3 illustrates the optimal transmit power versus the number of antennas selected under various circuit power consumption. The transmit power consumption increases with increase in number of selected antennas. The performance is evaluated for different circuit power consumption $\left(t_{\mathrm{c}}=30,40\right.$ and $\left.50 \mathrm{~mW}\right)$. The optimal transmit power increases when the circuit power consumption increases.

In Fig. 4, the SNR versus average data rate performance for the proposed antenna selection and power allocation with 128 antenna is compared with 64 antenna selected without power allocation. The proposed system gives better average data rate compared to 64 antenna configuration as the increase in the number of selected antennas improves the data rate of the system. More number of antennas used to suppress the high SNR to get the better average data rate.

\section{Conclusion}

In this paper, the antenna selection and power allocation is proposed for the Ma-MIMO system and the performance is evaluated. By activating all the antennas, the power consumed at the transmitter circuit increases which result in poor energy efficiency of the system. The antenna selection scheme reduced the number of RF chains used in the system resulting in low complexity and significant reduce in the power consumption. With this approach the sum rate maximization is achieved. Apart from the transmitter power consumption at the BS, various power consumption model is discussed in this paper. In order to reduce the total power consumption at the transmitter side, the optimal power allocation is combined with antenna selection to maximize the sum rate with reduced power consumption.

\section{References}

[1] LIU, Z., DU, W., SUN, D. Energy and spectral efficiency trade-off for Massive MIMO systems with transmit antenna selection. IEEE Transactions on Vehicular Technology, 2017, vol. 66, no. 5, p. 4453-4457. DOI: $10.1109 /$ TVT.2016.2598842

[2] HUANG, Y., HE, S., WANG, J., et al. Spectral and energy efficiency trade-off for Massive MIMO systems. IEEE Transactions on Vehicular Technology, 2018, vol. 67 , no. 8, p. $6991-7002$. DOI: $10.1109 /$ TVT.2018.2824311

[3] GAO, X., EDFORS, O., LIU, J., et al. Antenna selection in measured Massive MIMO channels using convex optimization. IEEE Globecom Workshops (GC Wkshps), 2013, p. 129-134. DOI: 10.1109/GLOCOMW.2013.6824974

[4] HANIF, M., YANG, H. C., BOUdREAU, G., et al. Antenna subset selection for Massive MIMO systems: A trace-based se- 
quential approach for sum rate maximization. Journal of Communications and Networks, 2018, vol. 20, no. 2, p. 144-155. DOI: $10.1109 / \mathrm{JCN} .2018 .000022$

[5] LI, H., GUO, J., WANG, Y., et al. Energy efficient antenna selection scheme for downlink Massive MIMO systems. In Proceedings of the IEEE International Symposium on Circuits and Systems (ISCAS). Florence (Italy), 2018, p. 1-4. DOI: 10.1109/ISCAS.2018.8351286

[6] QIAN, K., WANG, W. Q., SHAO, H. Low-complexity transmit antenna selection and beamforming for large-scale MIMO communications. International Journal of Antennas and Propagation, 2014, vol. 2014, 11 p. DOI: 10.1155/2014/159375

[7] HU, B. B., LIU, Y. A., et al. Energy efficiency of Massive MIMO wireless communication systems with antenna selection. The Journal of China Universities of Posts and Telecommunications, 2014, vol. 21, no. 6, p. 1-8. DOI: 10.1016/S1005-8885(14)60338-1

[8] ARASH, M., YAZDIAN, E., FAZEL, M. S., et al. Employing antenna selection to improve energy efficiency in Massive MIMO systems. Transactions on Emerging Telecommunications Technologies, 2017, vol. 28, no. 12. DOI: 10.1002/ett.3212

[9] ASAAD, S., BEREYHI, A., RABIEI, A. M., et al. Optimal transmit antenna selection for Massive MIMO wiretap channels. IEEE Journal on Selected Areas in Communications, 2018, vol. 36, no. 4, p. 817-828. DOI: 10.1109/JSAC.2018.2825159

[10] DONG, Y., TANG, Y., SHENZHEN, K. Z. Improved joint antenna selection and user scheduling for Massive MIMO systems. In Proceedings of the IEEE/ACIS 16th International Conference on Computer and Information Science (ICIS). Wuhan (China), 2017, p. 69-74. DOI: 10.1109/ICIS.2017.7959971

[11] ZHANG, J., WANG, J., WANG, Y. Antenna selection in Massive MIMO systems utilizing the submodular function. In Proceedings of the IEEE 9th International Conference on Wireless Communications and Signal Processing (WCSP). Nanjing (China), 2017, p. 1-6. DOI: 10.1109/WCSP.2017.8171056

[12] BENMimOUNE, M., DRIOUCH, E., AJIB, W., et al. Novel transmit antenna selection strategy for Massive MIMO downlink channel. Wireless Networks, 2017, vol. 23, no. 8, 2473-2484. DOI: $10.1007 / \mathrm{s} 11276-016-1297-9$

[13] LEE, B., NGO, L., SHIM, B. Antenna group selection based user scheduling for Massive MIMO systems. In Proceedings of the IEEE Global Communications Conference (GLOBECOM). Austin (USA), 2014, p. 3302-3307. DOI: 10.1109/GLOCOM.2014.7037316

[14] CASTANEdA, O., GOLdSTEIN, T., STUDER, C. POKEMON: A non-linear beamforming algorithm for 1-bit Massive MIMO. In Proceedings of the IEEE International Conference on Acoustics, Speech and Signal Processing (ICASSP). New Orleans (USA), 2017, p. 3464-3468. DOI: 10.1109/ICASSP.2017.7952800

[15] GAO, Y., VINCK, H., KAISER, T. Massive MIMO antenna selection: Switching architectures, capacity bounds, and optimal antenna selection algorithms. IEEE Transactions on Signal Processing, 2018, vol. 66, no. 5, p. 1346-1360. DOI: 10.1109/TSP.2017.2786220

[16] DU, L., LI, L., XU, Y. A genetic antenna selection algorithm with heuristic beamforming for Massive MIMO systems. In Proceedings of the IEEE 19th International Symposium on Wireless Personal Multimedia Communications (WPMC). Shenzhen (China), 2016, p. 49-52. DOI: 10.1109/RTUWO.2015.7365707

[17] SAKI, H., BAHAE, M. S. On the SINR distribution of SWIPT MUMIMO with antenna selection. arXiv preprint arXiv:1805.09463, 2018.

[18] BENMIMOUNE, M., DRIOUCH, E., AJIB, W., et al. Joint transmit antenna selection and user scheduling for Massive MIMO systems. In Proceedings of the IEEE Wireless Communications and Networking Conference (WCNC). New Orleans (USA), 2015, p. 381-386. DOI: $10.1109 /$ WCNC.2015.7127500

[19] KONAR, A., SIDIROPOULOS, N. D. Greed is good: Leveraging submodularity for antenna selection in Massive MIMO. In Proceedings of the 51st Asilomar Conference on Signals, Systems, and Computers. Pacific Grove (USA), 2017, p. 1522-1526. DOI: 10.1109/ACSSC.2017.8335611
[20] LEE, B. J., JU, S. L., KIM, N. I., et al. Enhanced transmit-antenna selection schemes for multiuser Massive MIMO systems. Wireless Communications and Mobile Computing, 2017, vol. 2017, p. 1-6. DOI: $10.1155 / 2017 / 3463950$

[21] FANG, B., QIAN, Z., SHAO, W., et al. RAISE: A new fast transmit antenna selection algorithm for Massive MIMO systems. Wireless Personal Communications, 2015, vol. 80, no. 3, p. 1147-1157. DOI: $10.1007 / \mathrm{s} 11277-014-2077-4$

[22] BEREYHI, A., ASAAD, S., SCHAEFER, R. F., et al. Iterative antenna selection for secrecy enhancement in Massive MIMO wiretap channels. In Proceedings of the IEEE 19th International Workshop on Signal Processing Advances in Wireless Communications (SPAWC). Kalamata (Greece), 2018. DOI: 10.1109/SPAWC.2018.8445912

[23] ABDULLAH, Z., TSIMENIDIS, C. C., JOHNSTON, M. Quantuminspired Tabu search algorithm for antenna selection in Massive MIMO systems. In Proceedings of the IEEE Wireless Communications and Networking Conference (WCNC). Barcelona (Spain), 2018, p. 1-6. DOI: 10.1109/WCNC.2018.8377099

[24] HUSBANDS, R., AHMED, Q., WANG, J. Transmit antenna selection for Massive MIMO: A Knapsack problem formulation. In Proceedings of the IEEE International Conference on Communications (ICC). Paris (France), 2017, p. 1-6. DOI: 10.1109/ICC.2017.7996694

[25] PARK, D. Transmit antenna selection in Massive MIMO systems. In Proceedings of the IEEE International Conference on Information and Communication Technology Convergence (ICTC). Jeju (South Korea), 2017, p. 542-544. DOI: 10.1109/ICTC.2017.8191036

[26] HAMDI, R., AJIB, W. Joint optimal number of RF chains and power allocation for downlink Massive MIMO systems. In Proceedings of the 82nd Vehicular Technology Conference (VTC Fall). Boston (USA), 2015, p. 1-5. DOI: 10.1109/VTCFall.2015.7391002

[27] TAI, T. H., CHUNG, W. H., LEE, T. S. A low complexity antenna selection algorithm for energy efficiency in Massive MIMO systems. In Proceedings of the IEEE International Conference on Data Science and Data Intensive Systems (DSDIS). Sydney (Australia), 2015, p. 284-289. DOI: 10.1109/DSDIS.2015.39

\section{About the Authors...}

Paranche Damodaran SELVAM (corresponding author) received B.E in Electronics and Communication Engineering from University of Madras, Chennai, India, M.E in Communication Systems from Anna University, Chennai, India and M.S in Mobile Communication from Institute Eurecom, Telecom ParisTeach, Sophia Antipolis, France, in 2002, 2007 and 2013 respectively. He has more than ten years of teaching experience in teaching for undergraduate students. He is currently pursuing Ph.D in Information and Communication Engineering from Anna University, Chennai, India. His research interest includes 5G, Massive MIMO, and Device to Device Communication.

Kuttathati Srinivasan VISHVAKSENAN received the MS degree from Birla Institute of Technology and Science, Pilani, Rajasthan, India in 1997. He received M.E degree and Ph.D degree from College of Engineering, Guindy, Anna University, Chennai, India in 2007 and 2013 respectively. $\mathrm{He}$ authored/ co-authored 30 IEEE International conferences and 20 international journals. His research field includes MIMO, iterative receiver algorithm, multi-user communications and transmitter preprocessing techniques, cooperative communication. 